Monatsschrift f. Geburtshülfe u. Gynäkologie 1933;95:I-IV

\title{
Contents, Vol. 95, 1933
}

\section{Inhalts verzeichni s.}

Oríginalarbeí́e $\pi$ seite

Abraham, Ernst G., Lassen sich hochfieberhafte Erkrankungen durch

My-Jod beeinflussen? 162

Agaronow, A. M., Über die Vereinfachung der Eeaktion Zondek-

Asch-heim und ihren praktischen Wert

20

Batisweiler, Joliann, Die Bedeutung des Scliüttelfrostes für die

Prognose des Wochenbettnebers 56

Chanina, F., siehe Weleshewa, A.

Danckwardt, L, Ein primäres doppelseitiges Tubenkarzinom .... 84

Denisenko, S. A., Zur Frage der präzisen Feststellung der Diagnose

bei Chorionepitheliom $\quad 381$

Eyding, A., Chorionepitheliom intra graviditatem unter dem klinischen

Bild einer Leukanaemia 867

Fuge, Kurt, Über einen Fall von verkalktem Myom während Schwanger-

schaft, Geburt und Wochenbett 66

Franqué, Otto v., Erkennung und Bekämpfung des Karzinoms. Be-

richtigung zu der Arbeit in Bd. 94, S. 255110

G a j z á g v́, E u g e n , Über eine Spođdylolisthese traumatischen Ursprungs 54 Gundlach, Y., siehe Schafer, W.

Haun, K., Beitrag zur Frage des Stumpfkarzinoms 91

Hauptmanu,Hans, ZurHistologiedesOvarsbeiMenstruationsanomalien 13 Hellner, Hans,

Experimentelle Untersuchui > gen zur amniogenen Ent-

stehung der Mißbildungen 70

Herberz, Oskar, Zur Kasuistik der Nabelendometriosen $\quad 259$

Horn, L·uise-Lotte, Ein Beitrag zur Atiologie des Wochenbettnebers 43

Joffe, T., Ileus und Sclrsvangerschaft 219

Kakuschkin, N., Beitrag zur Frage der Uterusperforation bei der Aus-

schabung 135

Kammerhuber, Fritz, Über intra partum entstehende Schädigungen

des Ileosakralgelenkes (an Hand eines beobachteten Falles) .... 378 Kotelnikoff, W., siehe

Weleshewa, A. Montag, Heinz, Achsendrehung des hochschwangeren Uterus mit

Placenta praevia und andere Anomalien bei asthenischen Ptoticae 359 Müller, Wilhelm, Über

Lungenernbolien nach gynäkologischen

Operationen 153

Ochssée, M. C. Boon v., Über die Anwendung des „Pernocton” beim

eklamptischen Anfall 227

Podleschka, Kurt, Klinisch-statistischeBeiträge zur Frage der Embolie 
Roessler, G., 1st eine Erweiterung der Aussichten und Grenzen für die Frühdiagnose des Gebärmutterkrebses möglich? 176

Schäfer, W., und V. Gundlach, Unsere Erfahrungen mit dem Wehenmittel Thymophysin 26

Schenk, F., 1st auf eine gesetzliche Regelung der eugenetisch indizierten

Schwangerschaftsunterbrechung Wert zu legen? 209

Scliepetinsky, A., Die Bildung einer ktinstlichen Scheide nach Baldwin 270

X"y Inhaitsverzeíchnis.

Seit $\beta$ Schulcz,Agostv., Kaiserschnitterfolge (152 Operatiouen ohne Todesfall) 144 - Die ßolle des Arztes und der Rechtspflege im Kampf gegen die

kriminelled Aborte 398

Schultz, Wo If hart, Ivorporale Blutungen im Greisenalter 76

Schultze, Kurt Walter, Holacardius amorphus globosus $\quad 389$

Ssolowjew, Wl., Uber die manuelle Plazentalösung 34

Szathmáry, Zoltán v., Über die konstitutionellen Unterschiede bei

ungarischen und germanischen Frauen, mit besonderer Berücksichtigung

der Weichteilverletzungen bei der Geburt und deren Folgen. ... 1

Weleshewa, A., W. Kotelnikoff, und F. Chan in a, Die Methodik

und Bedeutung der Therapie der spontanen Bupturen des Uterus-

kollum während der Geburt als Prophylaxe des geburtshilflichen

Trauma und seiner Folgen 129

Grenzgebíete der Geburtshilfe und Gynäkologie. Soziale Gynäkologie und Oeburtshilfe (III).

Von Fr. Chr. Geller, Breslau 96 Ko $\pi$ stitutionstherapie, unter besonderer Berücksichtigung der

Leibesiibungen.

Von Fritz Stähler, Frankfurt a. M 103

Physiologie und Krankheiten der Neugeborenen einschließlich derjenigen

der weiblichen Brust. (Jahresbericht III.) Von H.ßossenbeck, Gießen 179 Tuberkulose als

Grenzgebiet der Geburtshilfe und Gynäkologie (III). Von

Prof. Dr. F. Schultze-ßhonhof, Heidelberg, und Prof. Dr. K.

Han sen, Lübeck 274

Psychiatrie, Psychotherapie und Neurologie (III). Von Privatdozent Dr.

H. Siedentopf, Leipzig

409

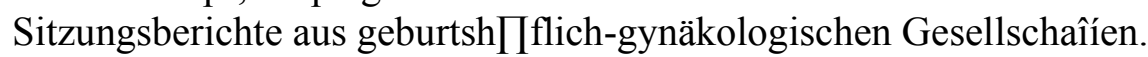

Gynäkologische Gesellschaft zu Breslau. Sitzung vom 28. März 1933 . . Ill Münchener

Gynäkologische Gesellschaft. Sitzungen vom 1. Dezember 1932

und 23. März 1933115

Geburtshilflich-gynäkologische Gesellschaft in $\Lambda$ Vien. Sitzungen vom

16. Mai und 13. Juni 1933121

Gesellschaft für Geburtshilfe und Gynäkologie zu Berlin. Sitzung vom

13. Januar 1933287

Xordwestdeutsche Gesellschaft für Gynäkologie. Sitzungen vom 13. und

14. Mai 1933 in Bad Pyrmont 298

Bayerische Gesellschaft für Geburtshilfe und Frauenheilkunde. Sitzung

vom 12. Februar 1933 in München 305

Streiflichter vom 57. Deutschen Chirurgenkongreß 416

Buchbesprechungen 124, 353, 416 
Perso $\pi$ alien $\quad 128,208,357,41$

OttoSarwey $\dagger 35$

A. Dührssen $\dagger$ 